\title{
Visualizar las redes de conocimiento: el caso Glass Cast
}

\author{
Ernesto Peña, Teresa Marie Dobson, Omar Juárez
}

\begin{abstract}
Resumen
El propósito de este artículo es presentar Glass Cast, una interfaz para la visualización de redes de conocimiento, la cual podría considerarse como un ejemplo del involucramiento de diseñadores profesionales desde la conceptualización de visualizaciones específicamente para humanidades digitales. La visualización de redes de conocimiento está basada en relaciones explícitas e implícitas entre documentos de una base de datos. Dichas relaciones están determinadas por parámetros tales como autoría, tiempo, tema, disciplina u otras, de acuerdo con el criterio y deseos del investigador. Los datos son adquiridos a partir de reportes creados dentro de Zotero, el conocido administrador de referencias libre. La interfaz funcional completa puede ser descargada desde el repositorio GitHub, aunque está abierta a cambios, adaptaciones y mejoras tanto por el equipo original de desarrollo como por cualquier otra parte interesada.
\end{abstract}

Palabras clave: visualización, humanidades digitales, interfaces, diseño de la información, redes de conocimiento, movilización del conocimiento.

\section{Visualize knowledge networks: the Glass Cast CASE}

\begin{abstract}
The purpose of this article is to introduce Glass Cast, an interface for the visualization of knowledge networks, which could be deemed as an example of the involvement of professional designers from the stage of conceptualization of visualizations, specifically intended for digital humanities. The visualization of knowledge networks is based on explicit and implicit relationships between documents in a database. These relationships are determined on parameters such as authorship, time, topic, discipline or others that would depend on the researcher' s criterion. The data is acquired from reports created from Zotero, the well-known open citation manager. The full working interface can be downloaded from GitHub although it is open to changes, adaptations and improvements either from the original development team or from any other interested party.
\end{abstract}

Keywords: visualization, digital humanities, interfaces, information design, knowledge networks, knowledge mobilization.

DOI: http://doi.org/10.22201/codeic.16076079e.2019.v20n3.a4 


\section{Ernesto Peña}

http://orcid.org/0000-0003-2254-954X

ernesto.pena@gmail.com sightation.com

ernestopena.com

Doctor en Filosofía en el Departamento de Educación en Alfabetización y Lenguaje por la Universidad de la Columbia Británica (University of British Columbia) en Vancouver, Canadá. Director de Investigación de Charitable Impact (chimp.net). Investigador independiente, diseñador y educador en diseño. Sus intereses de investigación incluyen diseño de la información, humanidades digitales, tipografía y alfabetización visual.

\section{Teresa Dobson}

http://blogs.ubc.ca/tmdobson/ teresa.dobson@ubc.ca

Profesora en el Departamento de Educación en Alfabetización y Lenguaje en Universidad de la Columbia Británica (University of British Columbia) en Vancouver, Canadá. Sus intereses de investigación incluyen educación en alfabetización y literatura, alfabetización digital y humanidades digitales. Su trabajo en humanidades digitales incluye colaboración con equipos académicos interdisciplinarios en el desarrollo de prototipos e interfaces para la visualización de textos.

\section{Omar Juárez}

www.omarojo.com omar@segartek.mx

Desarrollador de software y Máster en Medios Digitales por el Centro de Medios Digitales (Centre of Digital Media) en Vancouver, Canadá. Se especializa en el diseño y construcción de experiencias interactivas audiovisuales usando tecnologías web y protocolos de comunicación en tiempo real. Actualmente desarrolla software especializado para la transmisión de video broadcasting online con filtros y shaders desarrollados para ejecutarse directamente en el GPU de dispositivos móviles. 


\section{Introducción}

En los últimos años, la influencia de métodos visuales de análisis se ha vuelto más que evidente. Esta influencia se siente en prácticamente todos los campos del conocimiento y las Humanidades Digitales (HD) no son la excepción. Probablemente el signo más claro (y aún controversial) de la influencia de los medios visuales es el concepto de lectura distante de Moretti (2000). Viniendo de una tradición académica conservadora como lo es el análisis literario, Moretti propone distanciarse del texto lo suficiente como para percibir patrones generales, aunque eso signifique sacrificar el texto como unidad de análisis. En un artículo publicado posteriormente, el mismo autor presenta recursos como gráficos y visualizaciones como formas válidas de análisis (Moretti, 2003). Las implicaciones de la propuesta de Moretti van más allá de la implementación de nuevos métodos de análisis a la crítica literaria, también abren la puerta a investigadores expertos en métodos visuales a involucrarse en nuevas formas de producción de conocimiento y, más aún, invitan a la investigación interdisciplinaria (ver figura 1).

Figura 1. Representación gráfica de la popularización de la novela.

Reproducción tomada del trabajo original de Moretti

(2003).

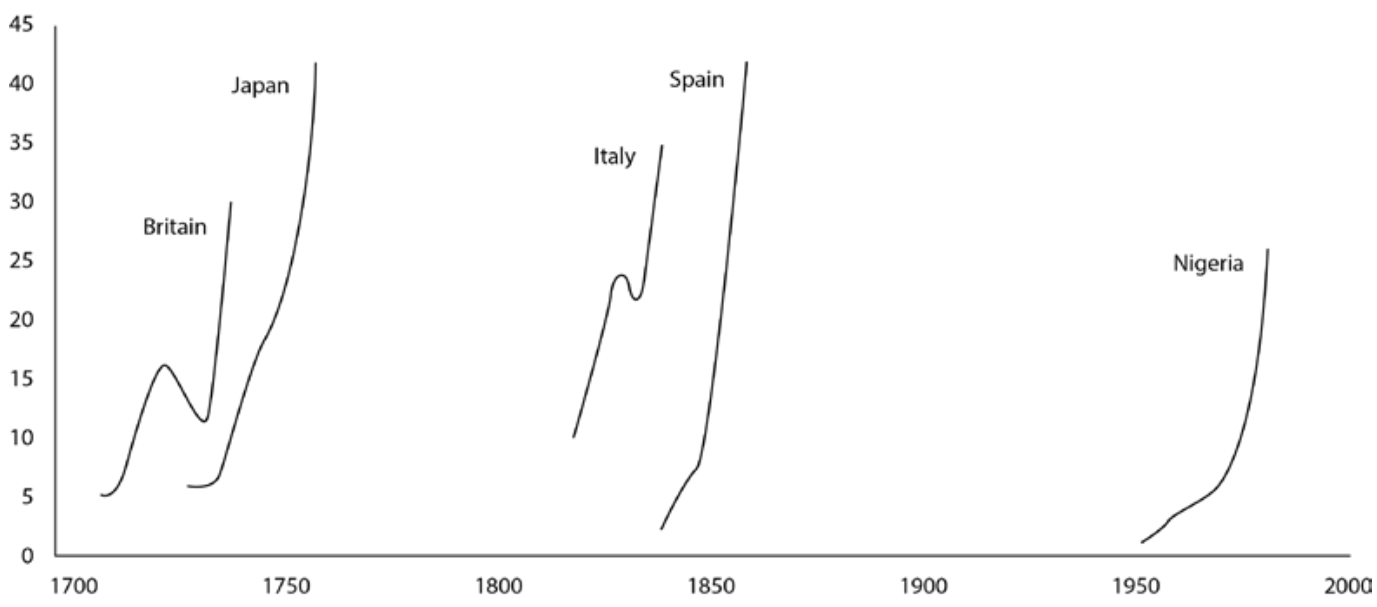

\section{El giro visual en Humanidades Digitales}

Mas que una disciplina per se, las HD son mejor definidas como un conjunto de prácticas. Entre ellas, la visualización de datos ${ }^{1}$ — la presentación de relaciones abstractas entre unidades de información por medios visuales - parece ser una de las que más atención ha recibido recientemente (Drucker, 2015). A este fenómeno se le ha llamado "el giro visual"2 en las HD (Burdick, Drucker, Lunenfeld, Presner, y Schnapp, 2012).

Es posible asumir que la necesidad de manejar y representar bases masivas de datos (un concepto habitualmente Ilamado Big Data) haya contribuido no sólo a la demanda de recursos de visualización, sino también al resurgimiento de campos de estudio como el diseño de la información, ${ }^{3}$ un campo del diseño que "involucra investigación de principios necesarios para el análisis, planeación,
1 Data visualization.

2 "The visual turn". 3 Information design. 
presentación y comprensión de mensajes —sus contenidos, lenguaje y forma" (Petterson, 2002, p. 2) , y que había perdido cierta relevancia desde mediados de la década pasada.

A su vez, el resurgimiento del diseño de la información ha llamado la atención de diseñadores e investigadores en diseño hacia las HD. Una muestra de esto es el último número del año 2015 de la revista académica Visible Language, dedicada precisamente a la relación entre el diseño y HD. Entre los investigadores que participan en el número 49.3 de Visible Language se encuentra Anne Burdick, quien junto con otros autores hizo un llamado explícito al involucramiento de humanistas digitales en procesos de diseño:

Sólo existirán visualizaciones diseñadas específicamente para atender las necesidades de comunicación de las humanidades si los humanistas se vuelven activamente comprometidos en su diseño (Burdick, Drucker, Lunenfeld, Presner, y Schnapp, 2012, p. 42). ${ }^{5}$

Afortunadamente, para cuando Burdick et al. hicieron este llamado, muchas interfaces en las que humanistas digitales participaron como diseñadores a nivel conceptual ya habían sido desarrolladas o estaban en desarrollo. Ejemplos recientes de esto son PlotVis (Dobson et al., 2011) e interfaces como Paper Drill, Wrkflux y Workflow, por mencionar algunos recursos generados con propósitos específicos entre 2011 y 2012 bajo el auspicio de Implementing New Knowledge Environments (INKE, inke.ca). Siendo ella misma una humanista digital, el llamado de Burdick (2012) parece tratar de encarar no una absoluta falta de recursos de visualización, sino una producción que se queda corta considerando la creciente demanda.

Otro escenario que ha sido explorado - aunque probablemente no en todo su potencial - es el involucramiento directo de diseñadores profesionales en la producción de visualizaciones de una forma relativamente institucionalizada o mejor aún, de universidades e institutos orientados al diseño. Casos como el de la misma Anne Burdick desde el Art Center College of Design, Stan Ruecker desde el Illinois Institute of Technology o Milena Radzikowska desde la Mount

${ }^{4}$ Traducción del autor:

[Information design (ID)] comprises research on the principles for analysis, planning, presentation and understanding of messages their contents, language and form.

${ }^{5}$ Traducción del autor: [...] visualizations designed to specifically address the communication needs of humanities research will only be created if humanists become actively engaged in their design. Royal University podrían servir como ejemplo de este escenario. Siendo uno de los autores de este artículo un diseñador profesional, nos gusta pensar que Glass Cast es tanto una respuesta al llamado de Burdick a la producción de interfaces específicamente para $\mathrm{HD}$, como una muestra de los resultados positivos de la participación de diseñadores profesionales en la conceptualización de plataformas dirigidas a resolver problemas al seno de las humanidades.

\section{Visualizar la interdisciplinariedad}

En los últimos años, instituciones académicas a lo largo del continente americano han mostrado un renovado interés en la interdisciplinariedad. Éste se manifiesta tanto en disponibilidad de recursos para la investigación interdisciplinar como en la generación de métodos y propuestas para la adopción de procedimientos 
Figura 2. Visualización de redes. Los nodos representan instancias (individuos, documentos, etcétera) y las líneas, relaciones (Grandjean, 2013).

Figura 3. Visualización de redes sobre proyección Mercator, desarrollada y presentada por Börner et al., 2012.

6 Knowledge Mobilization. 7 Uno de los recursos más utilizados para generación de visualizaciones de red es el software libre Gephi

(Bastian, Heymann, y Jacomy, 2009)

8 Maps of Science. ya existentes (p. ej. Arana et al., 2016; O’Brien, Marzano, y White, 2013; Rylance, 2015; Saldis et al., 2015). Específicamente en los campos de Ciencias Sociales y Humanidades en Norteamérica angloparlante (y de forma particular en Canadá), esta búsqueda por la interdisciplinariedad ha tomado el nombre de movilización del conocimiento, ${ }^{6}$ un concepto que refiere a la transmisión de conocimiento del investigador hacia sus pares dentro y fuera de una disciplina específica y del investigador hacia audiencias no expertas, ya sea para proporcionar acceso al conocimiento generado, o para influir la implementación de políticas (Levin, 2008, 2011). Este concepto sería de algún modo análogo al de comunicación científica propuesto por Martin y Rocha (2007), en el cual se hace una distinción entre diseminación (cuando ocurre entre pares), difusión (de expertos hacia audiencias instruidas aunque no sean de la misma disciplina) y divulgación del conocimiento (cuando se dirige a todo público).

Al igual que muchas otras relaciones abstractas, la noción de movilización o distribución de conocimiento se ha intentado representar visualmente por medio de patrones más o menos establecidos. Entre ellos se encuentra la llamada visualización de redes $^{7}$ (ver figura 2). Ejemplos de este patrón de representación aplicado a la movilización o distribución del conocimiento se pueden encontrar en los llamados mapas de ciencias ${ }^{8}$ (p. ej. Börner et al., 2012), representaciones gráficas de las conexiones entre paradigmas científicos en los que las unidades cuantificables suelen ser documentos académicos identificados por disciplina (ver figura 3).
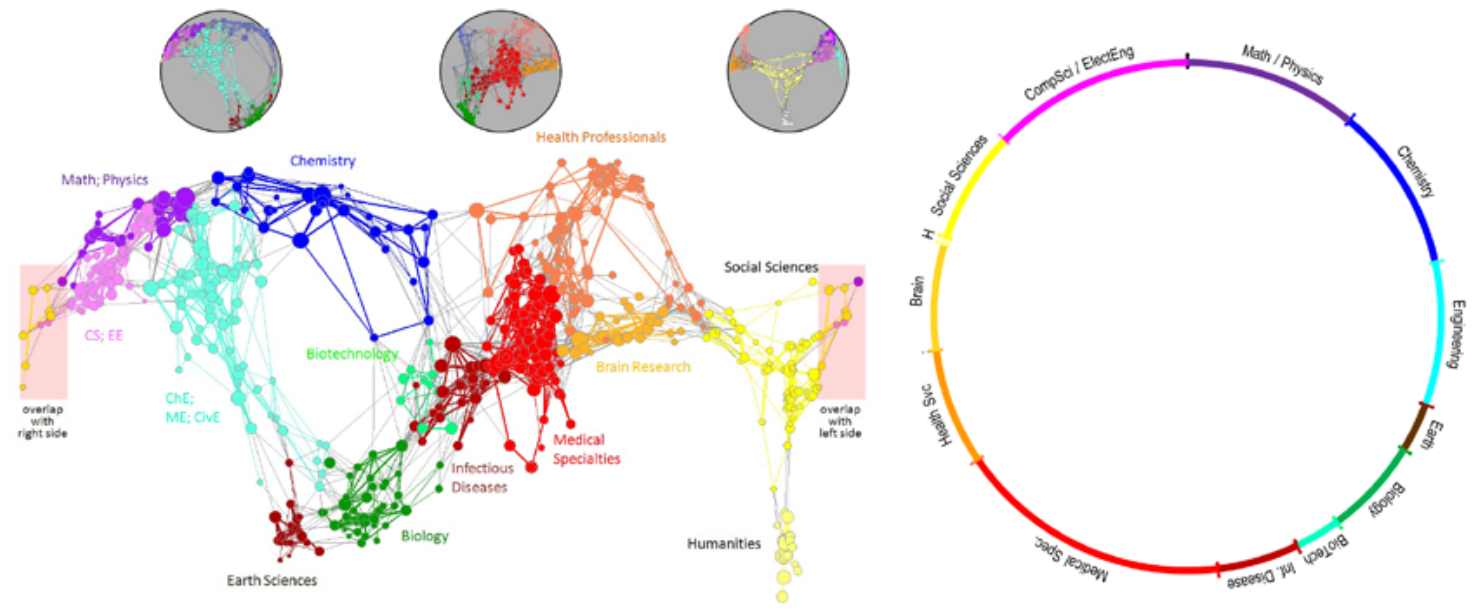

Aunque sumamente útiles y hasta cierto punto claros en la representación de relaciones, estos modelos muestran la transmisión de conocimiento como si sucediera en un plano temporal inmediato al investigador, obviando implícitamente los antecedentes históricos en su movilización (ver figura 4). El 
Figura 4. Los modelos actuales de movilización de conocimiento y comunicación científica representan al conocimiento desplazándose en un plano, en dos dimensiones. Estos modelos ignoran implícitamente su evolución histórica. Para nosotros, el modelo de la movilización del conocimiento es tridimensional. suponer que no es necesaria una revisión histórica en lo que respecta a la movilización del conocimiento sólo tendría sentido si estuviéramos seguros de que todo el conocimiento que se ha obtenido se ha abierto paso hasta el momento actual. Esta misma suposición se extiende hacia la comunicación científica y se ve reflejada en prácticas o argumentos de primera mano comunes en investigación académica, como el asumir que fuentes más recientes son intrínsecamente mejores. Los casos de investigación histórica son evidencia de que la movilización del conocimiento también ocurre en una dimensión temporal y que es necesario tomarlo en cuenta en su visualización.
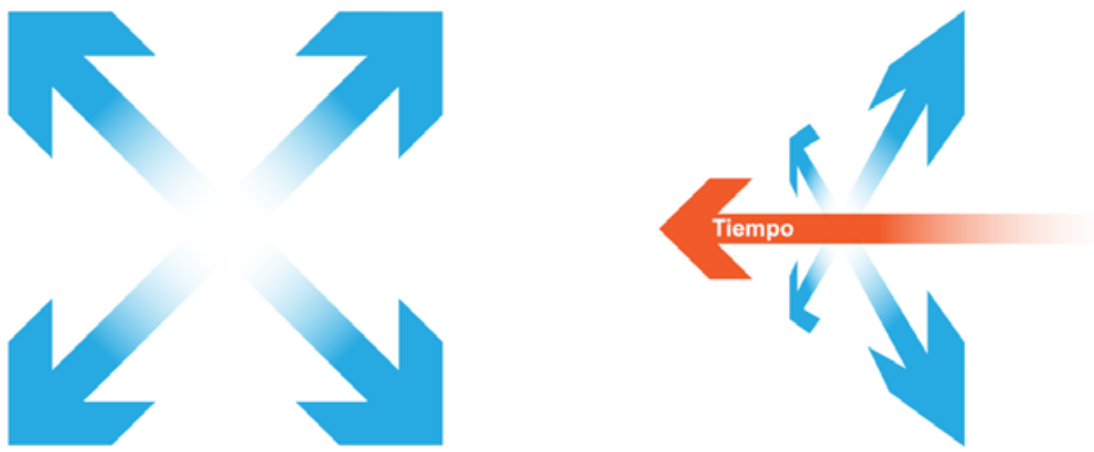

\section{Glass Cast}

Glass Cast surgió precisamente de la necesidad de hacer evidente la evolución que el término visual literacy ${ }^{9}$ ha sufrido al ser adoptado por diferentes disciplinas, al buscar mostrar las conexiones entre dichas disciplinas a lo largo de los años transcurridos desde su primera aparición en la literatura hasta nuestros días.

Existen diversas plataformas para facilitar la identificación de conexiones explícitas entre documentos (p. ej. Chen, 2004), muchas de ellas basadas en minería de datos y vinculación de referencias, un procedimiento que consiste en cosechar las referencias de un documento y sus metadatos. Sin embargo, el propósito de nuestra interfaz era considerar tanto las conexiones implícitas que no se reflejaran en las referencias de los documentos (p. ej., afiliación filosófica, relaciones de mentoría, etcétera), como las posibles implicaciones de la falta de conexiones. Particularmente en casos en los que la falta de referencia a un documento específico es un acto consciente y no una omisión circunstancial.

La plataforma debía poder adaptarse a las necesidades de cualquier investigador, ser relativamente sencilla de usar y estar disponible como software abierto. Algunas de estas premisas fueron atendidas al utilizar un administrador de referencias libre como base de datos. Zotero, a diferencia de otras aplicaciones destinadas al manejo de referencias bibliográficas, permite al investigador establecer relaciones entre documentos contenidos en una misma base de datos. Esta funcionalidad, aunada a la posibilidad de generar categorías

9 Entendido aquí como y notas para cada documento, le ofrece al investigador una interfaz familiar para "alfabetización visual". generación de redes de conocimiento. 
Figura 5. Aspecto de la interfaz de la plataforma Glass Cast abierta en una ventana de navegador. El investigador arrastra

el documento HTML del reporte generado por Zotero hacia el cuadro blanco al centro de dicha ventana.

10 Un proceso conocido como scraping.
Al haber sido conceptualizada como una plataforma para investigación histórica, fue necesario pensar en una manera de visualización cuya base fuera el patrón común de representación de redes de conocimiento, pero que además agregara a dicha visualización el tiempo de publicación de los documentos que conforman la base de datos. A raíz de una investigación sobre la facilidad con la que esta herramienta podría ser usada, para la cual se utilizaron modelos en papel (Peña y Dobson, 2015), se decidió distribuir los documentos de manera vertical sobre un eje y, de acuerdo con su fecha de publicación, y se generó así una visualización tridimensional. Las fechas establecerían la posición de los documentos sobre dicho eje y serían obtenidas directamente de la referencia de cada documento. Dado que Zotero genera reportes HTML de las bases de datos contenidas en su sistema, y que dichos reportes incluyen un listado de los documentos relacionados a cada ítem, fue posible establecer un protocolo de extracción de datos ${ }^{10}$ a partir de los cuales se generaría una visualización.

El resultado es una plataforma web que utiliza exclusivamente el reporte HTML generado desde Zotero para construir un modelo tridimensional de los documentos contenidos en la base de datos y sus interconexiones. El archivo del reporte es arrastrado hacia el centro de la ventana del navegador que contiene la página de la plataforma abierta (ver figura 5).

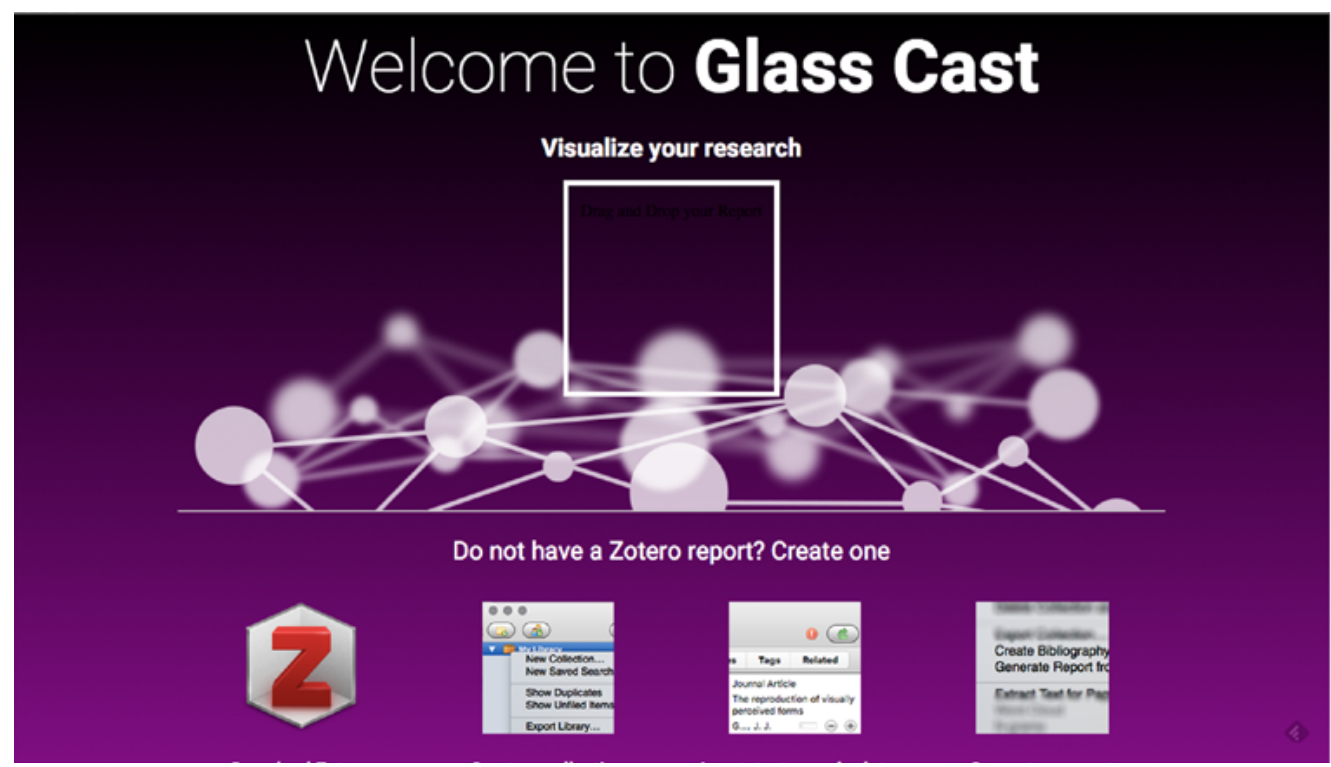

Los documentos y sus interconexiones son representados en un espacio tridimensional dentro del navegador de internet. Los documentos son simbolizados como esferas de diferente tamaño, dependiendo de la cantidad de conexiones con los que el documento cuente, las cuales son representadas con una línea. La posición de las esferas en el espacio tridimensional depende de la fecha de publicación de los documentos en la base de datos de Zotero. Los documentos son distribuidos sobre el eje y de los más recientes (arriba) a los más antiguos (abajo). Los colores de las esferas responden a categorías creadas por el mismo investigador desde la ventana Categorías ${ }^{11}$ de Zotero. El espacio 
11 Tags. tridimensional puede ser navegado utilizando las teclas W, A, S, D, Q, E, R, F y las flechas, permitiendo acercarse o alejarse a los documentos para observar mejor las conexiones y su origen (ver figura 6).

Figura 6. Aspecto de la interfaz de la plataforma Glass Cast después de integrar el reporte HTML de Zotero.

Figura 7. Documentos iluminados en color de acuerdo a la selección de categoría y subcategoría establecida por el investigador desde Zotero.

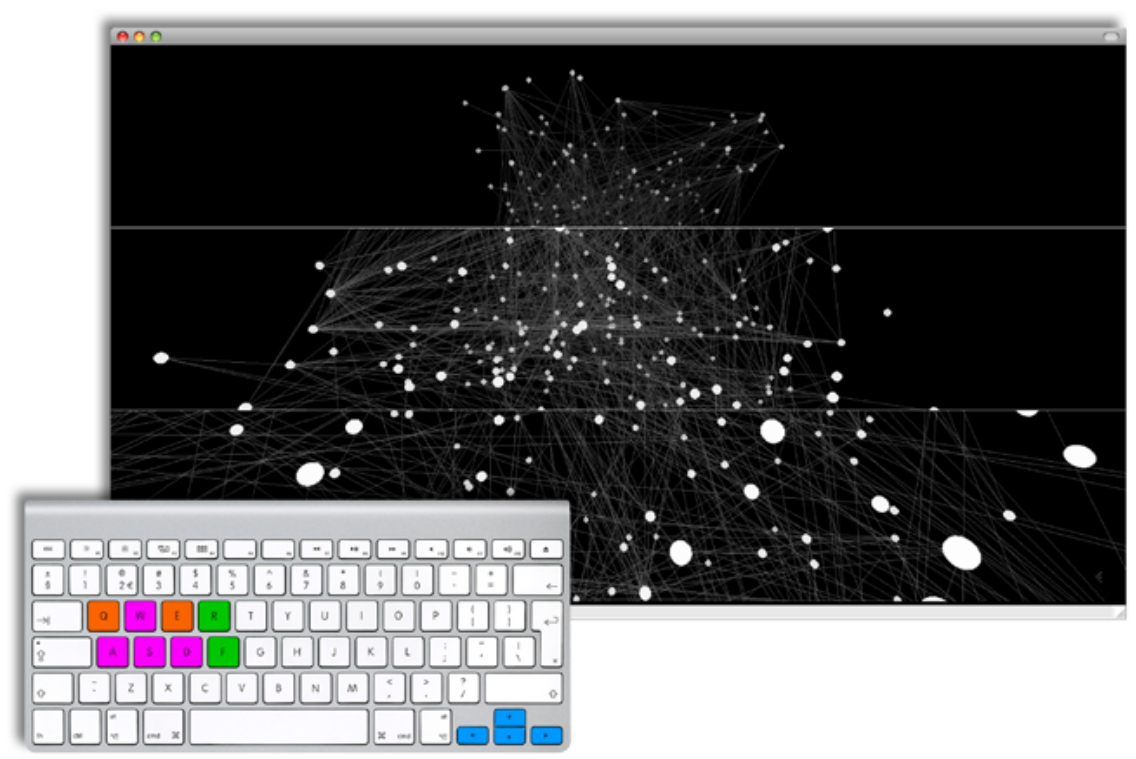

La interfaz cuenta, además, con paneles de información. Uno lateral que se activa al seleccionar un documento (representado en la interfaz como una esfera) y que muestra un listado de los documentos relacionados con él. El panel superior muestra un listado de categorías y subcategorías aplicadas por el investigador a cada documento desde Zotero. Los documentos en la interfaz se iluminan de diferente color dependiendo de la selección (ver figura 7).

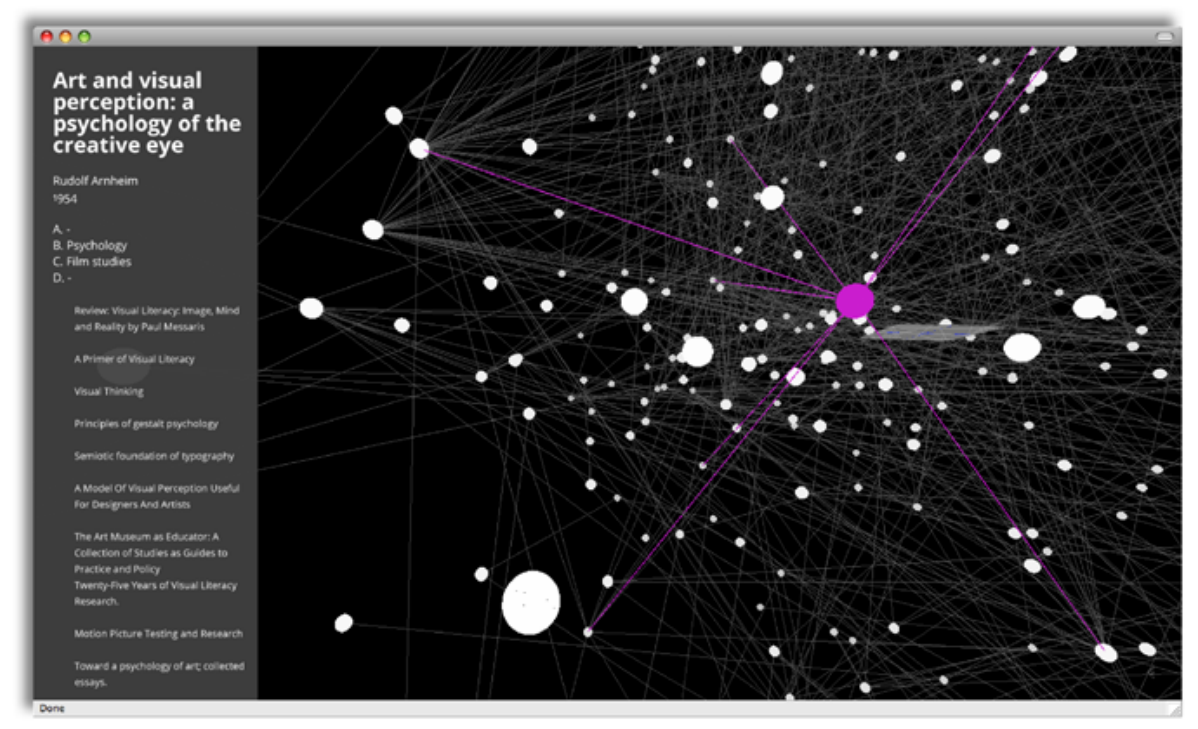

Glass Cast se conceptualizó como una forma de marco teórico visual para ser 
usado individualmente por investigadores a partir de bibliotecas personales. Esta aplicación permite dar un vistazo rápido a la distribución cronológica de los trabajos, la densidad de relaciones y otros tipos de afiliación entre los documentos que pueden ser difíciles de visualizar sólo desde el texto.

Si bien Glass Cast está disponible para su uso en su estado actual, no es nuestra intención considerarlo un producto terminado, sino como un proceso constante. Es una interfaz abierta a cambios, adaptaciones y mejoras tanto por el equipo original de desarrollo o cualquier otra persona interesada. Futuras mejoras podrían incluir cámaras ortogonales, la posibilidad de visualizar más de un reporte HTML a la vez y otros patrones de representación. La interfaz completa puede ser utilizada en línea desde http://omarojo.github.io/ZoteroGraph y descargada desde el repositorio GitHub para su modificación https://github.com/omarojo/ ZoteroGraph/tree/gh-pages. El desarrollo de este prototipo fue financiado por el grupo de investigación INKE de 2013 a 2016.

\section{Conclusiones}

Si bien es cierto que la presencia de las HD en México tiene relativamente poco tiempo de ser reconocida a nivel institucional, y que de algún modo los humanistas digitales mexicanos estamos repitiendo los patrones que otros países experimentaron durante su propio proceso de adopción de este conjunto de prácticas, también es cierto que estamos aprendiendo sumamente rápido, tanto de nuestras propias experiencias como las de otros. El potencial que tienen los humanistas digitales mexicanos de contribuir a la solución de problemas comunes en HD a través de herramientas de visualización de datos es notable. Por un lado, México tiene una historia de encuentros con personajes clave en el desarrollo del diseño de la información como disciplina (p. ej., Neurath, 2009), algunos de los cuales llegaron al punto de hacer escuela en nuestro país (p. ej., Bonsiepe, 2016). Por otro lado, el puente entre el diseño de la información y las HD es tan claro que la única consecuencia lógica es tomar la visualización de datos como bandera. El proyecto presentado aquí es sólo un caso de colaboración entre diseñadores y programadores mexicanos, académicos y humanistas digitales en otras disciplinas y otros países, uno de - esperemos- muchos más por venir.

\section{Referencias}

* Arana, L. L., Escobar, M. G., Rodríguez, A. S., Martínez, M. C. N., Rivero, R. M. M., y Rivero, B. R. (2016). La interdisciplinariedad: una necesidad contemporánea para favorecer el proceso de enseñanza aprendizaje. Medisur, 14(3), 320-327.

* Bastian, M., Heymann, S., y Jacomy, M. (2009). Gephi: An Open Source Software for Exploring and Manipulating Networks. Recuperado de: https://gephi.org/ publications/gephi-bastian-feb09.pdf. 
- Bonsiepe, G. (2016). Gui bonsiepe | design discourse. Recuperado de: http:// www.guibonsiepe.com/.

Börner, K., Klavans, R., Patek, M., Zoss, A. M., Biberstine, J. R., Light, R. P., ... Boyack, K. W. (2012). Design and Update of a Classification System: The UCSD Map of Science. PLOS ONE, 7(7), e39464. DOI: https://doi.org/10.1371/journal.pone.0039464.

* Burdick, A. (2015). Meta!Meta!Meta!: A Speculative Design Brief for the Digital Humanities. Visible Language, 49(3), 13.

* Burdick, A., Drucker, J., Lunenfeld, P., Presner, T., y Schnapp, J. (Eds.). (2012). Digital humanities. Cambridge, MA: MIT Press.

Chen, C. (2004). Searching for intellectual turning points: progressive knowledge domain visualization. Proceedings of the National Academy of Sciences of the United States of America, 101 Supp/ 1, 5303-5310. Dol: https://doi.org/10.1073/ pnas.0307513100.

Dobson, T., Michura, P., Ruecker, S., Brown, M., y Rodriguez, O. (2011). Interactive Visualizations of Plot in Fiction. Visible Language, 45(3), 169.

- Drucker,J.(2015). Graphical Approaches to the Digital Humanities. EnS. Schreibman, R. Siemens, \& J. Unsworth (Eds.), A New Companion to Digital Humanities (pp. 238250). John Wiley \& Sons, Dol: https://doi.org/10.1002/9781118680605.ch17.

* Grandjean, M. (2013). English: Graph representing the metadata of thousands of archive documents, documenting the social network of hundreds of League of Nations personals. Recuperado de: https://commons.wikimedia.org/wiki/File:Social Network Analysis Visualization.png.

- Implementing New Knowledge Environments (INKE) Interface. (s.f.). Recuperado de: http://inke.ca/projects/interface/.

* Levin, B. (2008). Thinking About Knowledge Mobilization. Presentado en el cCL/ SSHRC Invitation Seminar on Knowledge Mobilization, British Columbia. Recuperado de: http://www.oise.utoronto.ca/rspe/UserFiles/File/KM\%20paper\%20May\%20 Symposium\%20FINAL.pdf.

- Levin, B. (2011). Mobilising research knowledge in education. London Review of Education, 9(1), 15-26. Dol: https://doi.org/10.1080/14748460.2011.550431.

- Martin-Sampere, M., y Rocha, J. (2007). El papel de los científicos en la comunicación de la ciencia y la tecnología a la sociedad: Actitudes, Aptitudes e Implicaciones (1a. ed.). Madrid, España: Comunidad de Madrid y csic.

* Moretti, F. (2000). Conjectures on world literature. New Left Review, 1, 54-68.

Moretti, F. (2003). Graphs, Maps, Trees. New Left Review, 24, 67-93.

- Neurath, M. (2009). The transformer: principles of making isotype charts. London: Hyphen Press. 
- O'Brien, L., Marzano, M., y White, R. M. (2013). "Participatory interdisciplinarity": Towards the integration of disciplinary diversity with stakeholder engagement for new models of knowledge production. Science and Public Policy, 40(1), 51-61. Dol: https://doi.org/10.1093/scipol/scs120.

Peña, E., y Dobson, T. (2015). Glass, Paper, Scissors: Investigating the Metaphors of the Glass Cast through Paper Prototyping. Scholarly and Research Communication, 6(2). Recuperado de: http://src-online.ca/index.php/src/article/view/219.

- Pettersson, R. (2002). Information Design. An introduction. Philadelphia, PA, USA: John Benjamins Publishing Company. Recuperado de: http://site.ebrary.com/lib/ alltitles/docDetail.action?doclD=10023492.

* Rylance, R. (2015). Grant giving: global funders to focus on interdisciplinarity. Nature, 525(7569), 313-315. Dol: https://doi.org/10.1038/525313a.

- Saldis, N. E., Larrosa, N. B., Gómez, M. M., Marín, M. A., Penci, M. C., y López, A. G. (2015). Una propuesta metodológica para favorecer la interdisciplinariedad de contenidos científicos. Virtualidad, Educación y Ciencia, 6(10), 63-76.

\section{Cómo citar este artículo}

* Peña, Ernesto, Dobson, Teresa Marie y Juárez, Omar (2019). Visualizar las redes de conocimiento: el caso Glass Cast. Revista Digital Universitaria (RDU). Vol. 20, núm. 3 mayo-junio. Dol: http://doi.org/10.22201/codeic.16076079e.2019.v20n3.a4 . 